\title{
Glucose variability and inner retinal sensory neuropathy in persons with type 1 diabetes mellitus
}

MS Stem¹, GE Dunbar ${ }^{1}$, GR Jackson², S Farsiu³ R Pop-Busui ${ }^{4}$ and TW Gardner ${ }^{1,4}$

\begin{abstract}
Purpose To quantify early neuroretinal alterations in patients with type 1 diabetes mellitus (T1DM) and to assess whether glycemic variability contributes to alterations in neuroretinal structure or function. Methods Thirty patients with T1DM and 51 controls underwent comprehensive ophthalmic examination and assessment of retinal function or structure with frequency doubling perimetry (FDP), contrast sensitivity, dark adaptation, fundus photography, and optical coherence tomography (OCT). Diabetic participants wore a subcutaneous continuous glucose monitor for 5 days, from which makers of glycemic variability including the low blood glucose index (LGBI) and area under the curve (AUC) for hypoglycemia were derived. Results Sixteen patients had no diabetic retinopathy (DR), and 14 had mild or moderate DR. Log contrast sensitivity for the DM group was significantly reduced (mean $\pm \mathrm{SD}=1.63 \pm 0.06)$ compared with controls $(1.77 \pm 0.13, P<0.001)$. OCT analysis revealed that the inner temporal inner nuclear layer (INL) was thinner in patients with T1DM $(34.9 \pm 2.8 \mu \mathrm{m})$ compared with controls $(36.5 \pm 2.9 \mu \mathrm{m})(P=0.023)$, although this effect lost statistical significance after application of the Bonferroni correction for multiple comparisons. Both markers of glycemic variability, the AUC for hypoglycemia $(R=-0.458, P=0.006)$ and LGBI $(R=-0.473$, $P=0.004)$, were negatively correlated with inner temporal INL thickness.

Conclusions Patients with T1DM and no to moderate DR exhibit alterations in inner retinal structure and function. Increased glycemic variability correlates with retinal thinning on OCT imaging, suggesting that fluctuations in blood glucose may contribute to neurodegeneration.
\end{abstract}

Eye (2016) 30, 825-832; doi:10.1038/eye.2016.48; published online 1 April 2016

Introduction

Diabetes mellitus (DM) has reached epidemic proportions in the United States, and with this explosion in the prevalence of $\mathrm{DM}$, there has been a concomitant rise in the incidence of diabetic retinopathy (DR). ${ }^{1} \mathrm{DR}$ is classically characterized as a microvascular complication of diabetes because of its visible effects on the retinal vasculature (eg microaneurysms, cotton wool spots, and so on), but it is now recognized that diabetes has early, deleterious effects on neuroretinal structure and function. ${ }^{2}$ Specifically, measures of neuroretinal function such as microperimetry, ${ }^{3}$ frequency doubling perimetry (FDP) ${ }^{4-7}$ and contrast sensitivity ${ }^{8,9}$ reveal reduced retinal sensitivity in patients with diabetes and no to minimal DR compared with controls. Similarly, many studies have demonstrated structural alterations to the retina among patients with diabetes and minimal to no retinopathy, as evidenced by changes in foveal thickness, ${ }^{3,10}$ retinal nerve fiber layer (NFL) thickness, ${ }^{11,12}$ and inner retinal thickness. ${ }^{13,14}$ The damage to retinal structure and function that occurs from DM suggests that retinopathy is part of the systemic sensory neuropathy that affects other nerves during DM.

Understanding the earliest alterations to neuroretinal structure and function in patients with DM is instrumental to detect complications from DM at their earliest stages, which will allow for preventive and effective therapeutic intervention beyond the lowering of hemoglobin $\mathrm{A}_{1 \mathrm{c}}\left(\mathrm{HbA}_{1 \mathrm{c}}\right)$. Although pivotal studies in patients with type $1^{15}$ and type $2 \mathrm{DM}^{16}$ have demonstrated that the primary and most effective means of preventing diabetes-related complications is through intensive metabolic control, as documented by a lower $\mathrm{HbA}_{1 c}$, recent analyses
${ }^{1}$ Department of

Ophthalmology and Visual Sciences, University of Michigan, Ann Arbor, MI, USA

${ }^{2}$ Maculogix Inc., Hummelstown, PA, USA

${ }^{3}$ Department of Ophthalmology, Duke University Medical Center, Durham, NC, USA

${ }^{4}$ Department of Internal Medicine, Division of Metabolism,

Endocrinology and Diabetes, University of Michigan, Ann Arbor, MI, USA

Correspondence:

TW Gardner, University of Michigan, WK Kellogg Eye Center, 1000 Wall Street, Ann Arbor, MI 48105, USA Tel: +1 734232 8283; Fax: +1 7342328030 E-mail: tomwgard@umich. edu

Received: 12 October 2015 Accepted in revised form: 27 January 2016 Published online: 1 April 2016 
have called into question whether $\mathrm{HbA}_{1 \mathrm{c}}$ alone accurately captures all of the risk associated with the development of diabetes-related complications. ${ }^{17,18}$

Increased glucose variability may confer added risk to the development of diabetes complications in addition to the known deleterious effects of chronic hyperglycemia as measured by $\mathrm{HbA}_{1 \mathrm{c}} \cdot{ }^{19-22}$ For instance, an association between glucose variability and reduced heart rate variability, ${ }^{23}$ a sign of cardiac autonomic neuropathy, was recently reported in patients with type 1 diabetes mellitus (T1DM). Glucose variability was also involved in other cardiac parameters such as increased left ventricular mass index. ${ }^{24}$ Such relationships between high glucose variability and impaired cardiovascular structure or function suggest that reducing blood glucose variability may have a therapeutic benefit for patients with DM.

Taken together, the existing literature supports the idea that early DR includes a neurodegenerative component and that increased glucose variability may contribute to the development of complications from DM. However, previous studies have generally used a single test and have not provided a comprehensive assessment of retinal function in early DR. We hypothesized that T1DM affects primarily the inner retina. Therefore, we performed several tests of inner and outer retinal structure and function to define threshold effects of T1DM on the retina in a wellcharacterized cohort of subjects with T1DM phenotyped for multiple microvascular complications as part of the clinical trial NCT01170832. ${ }^{23}$ Furthermore, to better understand potential risk factors for these changes, we evaluated associations between measures of glucose variability and markers of abnormal retinal structure or function.

\section{Materials and methods}

The University of Michigan Institutional Review Board approved this protocol and all participants signed a written consent.

\section{Subjects}

We recruited two groups of patients: (1) adults with T1DM group and (2) healthy controls (control group). Inclusion criteria for the T1DM group were: T1DM as defined by the American Diabetes Association diagnostic criteria; ${ }^{25}$ duration of $\mathrm{DM} \geq 5$ years; age $18-65$ years old; and best-corrected visual acuity (BCVA) $\geq 20 / 30$ in the study eye. Inclusion criteria for the control group were: absence of DM; age $\geq 18$ years old; and BCVA $\geq 20 / 30$ in the study eye. Patients with T1DM were recruited from an ongoing observational study evaluating the natural history of myocardial dysfunction in T1DM and possible effects of glucose variability and were comprehensively phenotyped for other complications (NCT01170832). ${ }^{23}$
Exclusion criteria for all subjects included evidence of neuropathy or nephropathy; history of cardiovascular disease (including coronary artery disease, heart failure, arrhythmias, valvular disease, prior stroke, or hypertension); prior kidney, pancreas, or heart transplant; malignancy (with the exception of basal cell carcinoma); neurologic disease; history of substance abuse; presence or history of diabetic macular edema or a pre-existing ocular disease such as glaucoma; and refractive error $> \pm 6.00$ diopters (spherical equivalent).

\section{Analysis of retinal function}

Each participant underwent a battery of ophthalmic tests and procedures including: refraction with measurement of Early Treatment Diabetic Retinopathy Study (ETDRS) BCVA and a comprehensive ophthalmologic examination; FDP; contrast sensitivity; dark adaptation; OCT measurements of retinal layer thicknesses; and fundus photography. The right eye was used as the study eye, but if the right eye did not meet the inclusion criteria, the left eye was used. A blood sample was obtained from each patient to measure $\mathrm{HbA}_{1 \mathrm{c}}$.

The Matrix FDP (Carl Zeiss Meditec, Dublin, CA, USA) was used to measure retinal sensitivity using the 24-2 fullthreshold testing strategy as described by Jackson et al. ${ }^{4}$ The Matrix stimulus is a grating of vertically oriented white and black bars, the number of which appears to double when the bars undergo high-frequency counter phase flickering. We tested the non-study eye in each patient first to account for learning differences before testing the study eye. Results from the non-test eye were not used in the final analysis. Contrast sensitivity was assessed monocularly with the Pelli-Robson contrast sensitivity chart (Haag-Streit USA, Mason, $\mathrm{OH}, \mathrm{USA}$ ) at $1 \mathrm{~m}$ under standard overhead lighting conditions. Patients were asked to read progressively dimmer triplets of letters until at least two letters in a triplet were incorrectly read. The logarithmic contrast sensitivity score was determined by the preceding triplet of letters. We measured dark adaptation using the AdaptDx dark adaptometer (MacuLogix, Hummelstown, PA, USA) using the protocol described by Jackson et al. ${ }^{4}$ Each patient's study eye was exposed at the start of the test to a 2-ms $5.8 \times 10^{4}$ scotopic $\mathrm{cd} / \mathrm{m}^{2} \mathrm{sec}$ flash to bleach the photoreceptors. Then, subjects were shown subsequent lights of varying intensity at a point $5^{\circ}$ superior to the fovea. The end point was the time required for the patient to consistently report seeing a stimulus intensity of $5 \times 10^{-4} \mathrm{~cd} / \mathrm{m}^{2}$ (the rod intercept).

\section{Analysis of retinal structure}

The Spectralis optical coherence tomography (OCT) instrument (Heidelberg Engineering, Heidelberg, Germany) was used to obtain a $20^{\circ} \times 20^{\circ}$ cube scan centered on the fovea 
(97 sections, 512A-scans in each B-scan, and $3.87 \mu \mathrm{m}$ axial resolution). Macular cube scans were divided into nine ETDRS areas for further analysis. Briefly, this consisted of three concentric circles of 1,3 , and $6 \mathrm{~mm}$ in diameter. The two outermost circles were further subdivided into four quadrants, yielding a total of nine regions to analyze per scan. Within each of these nine areas, we calculated the thickness of the NFL, ganglion cell layer+inner plexiform layer (GCL+IPL), inner nuclear layer (INL), outer plexiform layer+outer nuclear layer (OPL+ONL), inner segment/ outer segment layer (IS/OS), and retinal pigment epithelial layer (RPE). We used Duke Optical Coherence Tomography Retinal Analysis Program (DOCTRAP) software to estimate the upper and lower boundaries of these seven layers ${ }^{26}$ using a semiautomatic protocol described in our previous publication. ${ }^{27}$

Fundus photographs (seven fields, $30^{\circ}$ in each field) were taken of both eyes according to standard ETDRS protocol using the Zeiss FF 450 plus fundus camera (Carl Zeiss Meditec, Dublin, CA, USA). These images were used to grade the DR severity of each eye as none, mild, or moderate. ${ }^{28}$

\section{Analysis of glucose variability}

The assessment of glucose variability in this cohort has been described previously by Jaiswal et al. ${ }^{23}$ Briefly, the continuous glucose monitoring (CGM) sensor iPro CGM System (Medtronic, Northridge, CA, USA) was used to measure and record subcutaneous glucose readings.
Measurements were obtained at 5-min intervals over a course of 5 days. Several indices of glucose variability were derived including measures of hypoglycemic stress such as the LBGI and area under the curve (AUC) for hypoglycemia (defined as blood glucose $<70 \mathrm{mg} / \mathrm{dl}$ ) as described previously. ${ }^{29}$ In cases where CGM data were missing ( $~ 5 \%$ of data had gaps), the lost values were interpolated based on the adjacent observed values.

\section{Statistical analysis}

Data analysis was performed using SPSS Statistics (version 21.0; SPSS Inc., Chicago, IL, USA). Means and standard deviations were calculated for continuous variables, and frequencies and percentages are displayed for categorical variables. Normality was assessed for each variable using the Shapiro-Wilk test. Parametric tests were used to compare normally distributed variables, and nonparametric tests were used to evaluate non-normally distributed variables. Multiple regression analysis was performed to assess the relationship between measures of glucose variability and covariates such as age and results from retinal structure/function testing. The Bonferroni correction for multiple comparisons was applied to the OCT data such that a statistically significant result would occur at a $P$-value $\leq 0.0056$. For all other tests, $P \leq 0.05$ was considered statistically significant.

Table 1 Participant demographics

\begin{tabular}{|c|c|c|c|c|}
\hline & $T 1 D M$ & Controls (functional tests) & Controls (OCT) & P-value \\
\hline Participants, number & 30 & 23 & 50 & \\
\hline Sex & & & & $P=0.091^{\mathrm{a}}$ \\
\hline Male, $n(\%)$ & $10(33)$ & $13(57)$ & $22(44)$ & \\
\hline Female, $n(\%)$ & $20(67)$ & $10(43)$ & $28(56)$ & \\
\hline Age (years) (SD) & $38(13)$ & $48(20)$ & $51(17)$ & $P=0.028^{\mathrm{b}}$ \\
\hline HbA1c (\%) (SD) & $7.9(1.0)$ & $5.5(0.3)$ & - & $P<0.001^{\mathrm{b}}$ \\
\hline $\mathrm{mmol} / \mathrm{mol}$ (SD) & $63(10)$ & $37(4)$ & & \\
\hline Diabetes duration (years) (SD) & $14(6.7)$ & - & - & \\
\hline Systolic BP (mm Hg) (SD) & $125(14)$ & $119(15)$ & - & $P=0.152^{\mathrm{b}}$ \\
\hline Diastolic BP (mm Hg) (SD) & $74(9)$ & $67(8)$ & - & $P=0.004^{\mathrm{b}}$ \\
\hline BMI (SD) & $26.4(3.9)$ & $25.7(6.3)$ & - & $P=0.184^{c}$ \\
\hline Visual acuity, logMAR (Snellen equivalent) & $0.02(20 / 20)$ & $-0.06(20 / 15)$ & - & $P=0.004^{c}$ \\
\hline Retinopathy status & & - & - & \\
\hline No DR, $n(\%)$ & $16(53)$ & & & \\
\hline Mild DR, $n(\%)$ & $11(37)$ & & & \\
\hline Moderate DR, $n(\%)$ & $3(10)$ & & & \\
\hline
\end{tabular}

Abbreviations: BMI, body mass index; BP, blood pressure; DR, diabetic retinopathy; HbA1c, hemoglobin A1c; OCT, optical coherence tomography; SD, standard deviation; T1DM, type 1 diabetes mellitus.

Statistical tests compare the T1DM group vs Controls (functional tests).

${ }^{\mathrm{a}} \chi^{2}$ Test.

${ }^{\mathrm{b}}$ Independent-samples $t$-test.

${ }^{c}$ Mann-Whitney U-test. 


\section{Results}

Participant characteristics are listed in Table 1. We enrolled 30 patients with T1DM and 23 age-matched controls for functional testing (log contrast sensitivity, FDP, and so on). All of the participants were phakic with minimal to no cataract. Approximately half $(53 \%, n=16)$ of the patients with T1DM had no evidence of DR on fundus photography, and the other $47 \%$ had mild-tomoderate non-proliferative DR. In addition, OCT data from 28 additional controls were included in the analysis of retinal structure.

\section{Retinal function}

Table 2 depicts the results of the functional tests. In general, patients with T1DM exhibited reduced inner retinal function compared with controls, with relative preservation of outer retinal function. Specifically, log contrast sensitivity for the T1DM group was significantly reduced (mean $\pm \mathrm{SD}=1.63 \pm 0.06$ ) compared with controls $(1.77 \pm 0.13, P<0.001)$. Similarly, the mean deviation of the FDP was impaired in patients with T1DM $(-1.34 \pm 2.84 \mathrm{~dB})$ relative to controls $(0.57 \pm 2.49 \mathrm{~dB}$, $P=0.033)$. The time to dark adaptation, as measured by the rod intercept, was not significantly different between the two groups $(P=0.145)$, suggesting that photoreceptor/pigmented epithelial complex function was intact.

After stratifying participants with T1DM into two groups (with $(n=16)$ or without $(n=14)$ retinopathy), we found abnormalities in tests of inner retinal function among the groups. The mean deviation of the FDP was significantly reduced in patients with DR $(-2.77 \pm 2.68 \mathrm{~dB})$ compared with both the no DR group $(-0.02 \pm 2.36 \mathrm{~dB})$ and the control group $(0.57 \pm 2.49 \mathrm{~dB})(P=0.012)$. Log contrast sensitivity was similar between the DR $(1.63 \pm 0.05)$ and no DR $(1.64 \pm 0.07)$ groups, but both were significantly reduced compared with the control group $(1.77 \pm 0.13)(P=0.001)$.

\section{Retinal structure}

Figure 1 shows the results of the OCT analysis. The main finding is that in several areas of the macula, the INL was consistently thinner among patients with T1DM compared with controls. Specifically, the inner temporal INL was thinner in patients with T1DM $(34.9 \pm 2.8 \mu \mathrm{m})$ compared with controls $(36.5 \pm 2.9 \mu \mathrm{m})(P=0.023)$, as were the inner nasal INL $(37.1 \pm 2.9$ vs $38.5 \pm 2.7 \mu \mathrm{m}, P=0.044)$ and inner inferior INL $(38.0 \pm 3.4$ vs $39.6 \pm 2.7 \mu \mathrm{m}, P=0.04)$. However, after applying the Bonferroni correction for multiple comparisons, we did not find any statistically significant differences between the groups.

After stratifying the patients with T1DM into those with $(n=16)$ or without $(n=14)$ retinal vascular lesions, we did not find any statistically significant differences in INL thickness among controls, patients with T1DM and no DR, and those with T1DM and mild-to-moderate DR $(P=0.073$ for inner temporal INL, 0.117 for inner nasal INL, and 0.088 for inner inferior INL).

\section{Comparison of retinal structure and function}

After controlling for the effects of age using a multiple regression analysis, we found that higher log contrast sensitivity was significantly associated with a thicker (more intact) inner temporal INL value $(P=0.002$, $\left.\beta=0.475, R^{2}=0.224\right)$. FDP mean deviation, however, was not found to be predictive of inner temporal INL after controlling for age $(P=0.241)$.

\section{Glucose variability}

We correlated the two metrics of glucose variability (AUC for hypoglycemia and low blood glucose index (LGBI)) with tests of inner retinal function (FDP mean deviation and $\log$ contrast sensitivity) and structure (inner temporal INL thickness, as this region was thinnest among patients with diabetes relative to controls). Neither AUC for hypoglycemia nor LGBI were significantly correlated with FDP mean deviation, although there was a trend

Table 2 Functional testing

\begin{tabular}{|c|c|c|c|c|}
\hline Ocular test & T1DM & Controls & P-value & $\begin{array}{c}\% \text { of } T 1 D M \text { falling below } \\
\text { normal reference range }\end{array}$ \\
\hline FDP, MD & $-1.34(2.84 \mathrm{~dB})$ & $0.57(2.49 \mathrm{~dB})$ & $P=0.033$ & 18.5 \\
\hline FDP, PSD & $2.96(0.95 \mathrm{~dB})$ & $2.84(1.67 \mathrm{~dB})$ & $P=0.016$ & 0 \\
\hline FDP, foveal threshold & $29.24(4.93 \mathrm{~dB})$ & $30.77(3.62 \mathrm{~dB})$ & $P=0.420$ & 10.7 \\
\hline Log contrast sensitivity & $1.63(0.06)$ & $1.77(0.13)$ & $P<0.001$ & 14.2 \\
\hline Dark adaptation, rod intercept & 9.7 (1.0 min) & $9.0(1.5 \mathrm{~min})$ & $P=0.145$ & 3.7 \\
\hline
\end{tabular}

Abbreviations: FDP, frequency doubling perimetry; MD, mean deviation; PSD, pattern standard deviation; T1DM, type 1 diabetes mellitus.

Results of functional tests are displayed. Participants with diabetes tended to perform more poorly on tests of inner retinal function compared with controls. Comparisons were made with the Mann-Whitney $U$-test, and results are presented as mean (SD). The normal reference range was defined by the mean of the control group \pm 2 SDs. 

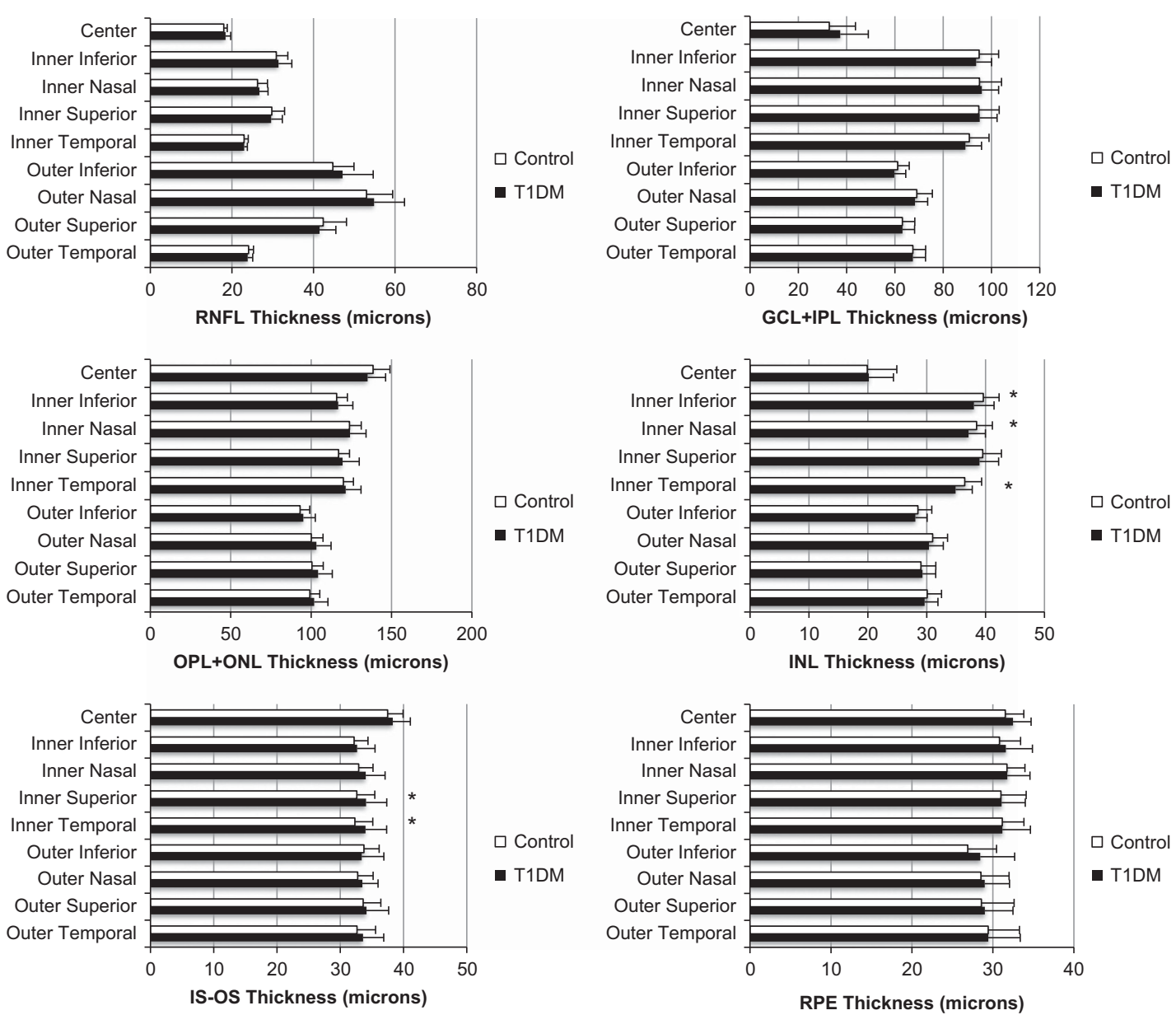

Figure 1 Retinal layer thicknesses were measured in a semiautomated manner from OCT images at each of nine areas in the macula of the right eye of each participant. Results are presented as mean \pm 1 standard deviation. Abbreviations: RNFL, retinal nerve fiber layer; IS-OS, inner segment outer segment; OPL+ONL, outer plexiform layer and outer nuclear layer; GCL+IPL, ganglion cell layer and inner plexiform layer; RPE, retinal pigment epithelium ( ${ }^{*} P<0.05$ vs control).

for both AUC $(R=0.297, P=0.088)$ and LGBI $(R=-0.312$, $P=0.073$ ) to be negatively correlated with log contrast sensitivity. That is, as measures of glucose variability and low blood glucose values increased, patients tended to do more poorly on the log contrast sensitivity test.

Regarding retinal structure, both AUC for hypoglycemia $(R=-0.458, P=0.006)$ and LGBI $(R=-0.473, P=0.004)$ were negatively correlated with inner temporal INL thickness (Figure 2). These relationships persisted after adjusting for age and recent $\mathrm{HbA}_{1 \mathrm{c}}$ values in multiple regression analyses. For AUC hypoglycemia, the $R^{2}$ value for the model was 0.264 with a $\beta$ of -0.445 and an overall $P$-value of 0.022 . For LGBI, the $R^{2}$ value for the model was 0.281 with a $\beta$ of -0.472 and an overall $P$-value of 0.015 .

While patients with DR had a slightly higher average recent $\mathrm{HbA}_{1 \mathrm{c}}$ compared with individuals without DR $(8.3 \pm 1.2 \%$ vs $7.6 \pm 0.7 \%, P=0.05)$, there was no difference in either AUC hypoglycemia or LGBI between individuals with and without DR. Similarly, there was no difference in the

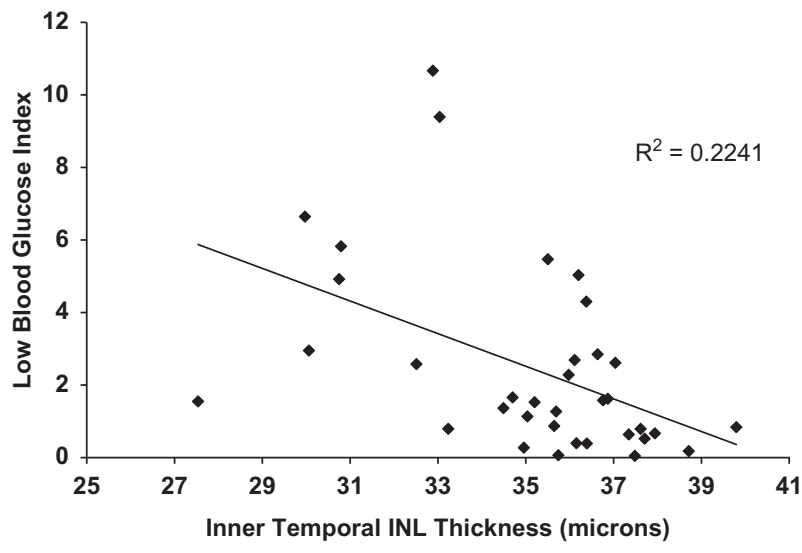

Figure 2 The LGBI, a measure of glucose variability, was negatively correlated with the thickness of the inner temporal INL (as measured with OCT). INL, inner nuclear layer. 
duration of DM between those with DR (13.2 \pm 6.8 years) and individuals without DR $(14.6 \pm 6.7$ years, $P=0.57)$. Diabetes duration was not significantly correlated with FDP mean deviation, inner temporal INL thickness, or log contrast sensitivity.

\section{Discussion}

In this study comparing individuals without diabetes and those with T1DM and minimal to no systemic complications, we found alterations in both retinal structure and function that localize to the inner retina. Notably, the patients with T1DM were extensively phenotyped by an endocrinologist (RP-B) and were overall in excellent health, despite some having subtle evidence of autonomic dysfunction. ${ }^{23}$ Matrix FDP sensitivity and $\log$ contrast sensitivity were reduced in patients with T1DM compared with controls. In addition, INL thickness was less in patients with DM relative to controls, which correlated with abnormalities seen on functional testing. Finally, markers of increased glucose variability such as LGBI and AUC hypoglycemia were correlated with reduced INL thickness, and this relationship persisted even after accounting for the effects of age and recent $\mathrm{HbA}_{1 \mathrm{c}}$ measurements.

Our finding that individuals with T1DM and moderate to no retinopathy have reduced FDP sensitivity, log contrast sensitivity, and INL thickness on OCT relative to controls supports the notion that diabetes has a predilection for perturbing inner retinal structure and function early in the course of the disease. Dark adaptation, a measure of outer retinal function, was not significantly impaired in the diabetic cohort relative to controls. In a 2012 report, Jackson et al ${ }^{4}$ also failed to find a significant impairment in dark adaptation among patients with diabetes relative to controls, although in a subgroup analysis they found that $26 \%$ of patients with NPDR exhibited rod intercept times that were outside the normal reference range. ${ }^{4}$ However, unlike our cohort comprised mainly of individuals with no to mild DR, they included many more participants with moderate to severe NPDR, which may explain why they found a greater percentage of patients with abnormal dark adaptation times. While we did not find any association with markers of increased glucose variability and neuroretinal dysfunction (as measured by FDP and contrast sensitivity), we did find a relationship between neurodegeneration in the INL of the retina and increased glucose variability, independent of the effects of $\mathrm{HbA}_{1 \mathrm{c}}$. To the best of our knowledge, this is the first report demonstrating a potential link between retinal structure and glucose variability.

Importantly, our findings corroborate the results from prior studies that have demonstrated reduced measures of inner retinal function among patients with $\mathrm{DM}^{4,5,8}$ and suggest that FDP sensitivity and/or contrast sensitivity may have potential roles as quantitative measures of neuroretinal dysfunction in patients with DM. Indeed, Scott et $a l^{30}$ recently used FDP as a clinical trial end point to evaluate the efficacy of doxycycline in preserving retinal sensitivity during diabetes. They found that doxycycline treatment was associated with improved FDP sensitivity at the end of the 24-month trial, although further work is needed to understand the clinical significance of improvements in FDP sensitivity.

Several studies have measured retinal layer thickness in patients with DM with various results. van Dijk and co-workers ${ }^{13,14,31}$ have consistently found that specific inner retinal layers, namely the ganglion cell, inner plexiform, and INLs, are thinner among patients with DM relative to controls. Similarly, other investigators have found reductions in NFL thickness in patients with DM, especially in the peripapillary area. ${ }^{11,12}$ In comparing total retinal thickness between patients with DM and controls, some studies have found patients with DM to have increased foveal thickness, ${ }^{3}$ others have found no difference, ${ }^{32-34}$ and others have found overall thinning of the retina. ${ }^{35}$ Despite these disparate results, it appears that most studies, regardless of instrumentation or method of segmentation, can localize retinal thinning to the inner retina when specific macular regions other than the fovea are measured. Although we did not find evidence of ganglion cell or NFL thinning in our cohort, our finding of INL thinning in patients with DM is consistent with a report from other investigators ${ }^{13}$ and correlates with the abnormalities we found on functional testing. While our OCT findings did not maintain statistical significance after application of the Bonferroni correction for multiple comparisons, they suggest a trend that merits further investigation with a larger sample size. Our segmentation analysis combined the ganglion cell and inner plexiform layer into a single measurement, whereas van Dijk et al ${ }^{14}$ attempted to split these layers into individual measurements. This difference in methodology, combined with our smaller cohort size, may explain our inability to replicate their findings of decreased perifoveal ganglion cell layer thickness in patients with DM.

Although $\mathrm{HbA}_{1 \mathrm{c}}$ remains the central metric of glucose control and an important prognostic factor for the development of diabetes complications, recent reports have called into question whether $\mathrm{HbA}_{1 \mathrm{c}}$ alone may predict all individuals at risk for developing complications from diabetes. ${ }^{17,20}$ Fluctuations in blood glucose may have an equally important role in predisposing individuals to the adverse outcomes associated with prolonged diabetes. ${ }^{22,23}$ The emergence of new technology to quantify neuroretinal dysfunction and degeneration may provide an opportunity to test the hypothesis that glucose variability, in addition to 
sustained hyperglycemia, ${ }^{36}$ contributes to end-organ damage from diabetes in the eye. Our finding that thinning of the INL was associated with high glucose variability complements the recent report by Jaiswal et $a^{23}$ describing decreased heart rate variability as a result of dysfunction in peripheral autonomic nerves among patients with high levels of glucose variability. Taken together, these and other studies ${ }^{21,24}$ suggest that glucose variability, in addition to the deleterious effects of chronic hyperglycemia, may contribute to end-organ damage in patients with DM.

This was a small, cross-sectional study and thus precludes the drawing of definitive conclusions about the relationship between glucose variability and retinal structure or function. The main strength of the study is that it evaluated an extensively characterized (both in terms of eye health and systemic health) group of participants. Furthermore, to the best of our knowledge, this is the first report that attempts to correlate measures of glucose variability with metrics of retinal structure or function.

The classic notion of DR as a microvascular complication of diabetes has been called into question by accumulating evidence suggesting that neurodegeneration occurs in the diabetic retina. Emerging technologies such as the matrix FDP and spectral domain OCT have in many studies localized early alterations to neuroretinal function and structure to cells of the inner retina. With these quantitative tools, it is easier to assess subtle changes in retinal physiology to better understand what factors contribute most to neuroretinal dysfunction during diabetes. Although the contribution of increased glucose variability to the pathogenesis of diabetic complications remains complex, this and other studies suggest that short-term fluctuations in blood glucose may have a negative impact on end organs that are targeted by diabetes. These negative effects seem to persist even after accounting for the role of $\mathrm{HbA}_{1 \mathrm{c}}$, suggesting that glucose variability may have a supplementary role to chronic hyperglycemia in the development of complications from diabetes. As CGM becomes more widespread and the technology for assessing retinal physiology improves, it will be important to continue to evaluate the relationship between blood glucose fluctuations and neuroretinal health.

\section{Summary}

What was known before

- Diabetes has deleterious effects on neuroretinal health.

What this study adds

- Diabetes has a predilection for disrupting inner retinal structure and function.

- Glycemic variability is associated with thinning of the INL of the retina, suggesting that fluctuations in blood glucose levels might be associated with neurodegeneration in patients with diabetes.

\section{Conflict of interest}

The authors declare no conflict of interest. The authors alone are responsible for the content and writing of the paper.

\section{Acknowledgements}

This work was financially supported by American Diabetes Association-Merck Clinical/Translational Postdoctoral Fellowship Award (to TWG and MSS); JDRF and A Alfred Taubman Medical Research Institute Healthy Eyes Scholar Award (to TWG); Research to Prevent Blindness Physician-Scientist Award, EY20582 and DK094292 (to TWG); National Institutes of Health/National Heart, Lung, and Blood Institute R01-HL-102334 (to RP-B); American Diabetes Association Grant 1-14-MN-02 (to RP-B); NIH R01 EY022691 (to SF); NEI P30 EY-005722 (to SF).

\section{References}

1 Saaddine JB, Honeycutt AA, Narayan KM, Zhang X, Klein R, Boyle JP. Projection of diabetic retinopathy and other major eye diseases among people with diabetes mellitus: United States, 2005-2050. Arch Ophthalmol 2008; 126(12): 1740-1747.

2 Simo R, Hernandez C. Neurodegeneration is an early event in diabetic retinopathy: therapeutic implications. Br J Ophthalmol 2012; 96(10): 1285-1290.

3 De Benedetto U, Querques G, Lattanzio R, Borrelli E, Triolo G, Maestranzi $\mathrm{G}$ et al. Macular dysfunction is common in both type 1 and type 2 diabetic patients without macular edema. Retina 2014; 34(11): 2171-2177.

4 Jackson GR, Scott IU, Quillen DA, Walter LE, Gardner TW. Inner retinal visual dysfunction is a sensitive marker of nonproliferative diabetic retinopathy. Br J Ophthalmol 2012; 96 (5): 699-703.

5 Parravano M, Oddone F, Mineo D, Centofanti M, Borboni P, Lauro $\mathrm{R}$ et al. The role of Humphrey Matrix testing in the early diagnosis of retinopathy in type 1 diabetes. $\mathrm{Br} J$ Ophthalmol 2008; 92(12): 1656-1660.

6 Parikh R, Naik M, Mathai A, Kuriakose T, Muliyil J, Thomas $\mathrm{R}$. Role of frequency doubling technology perimetry in screening of diabetic retinopathy. Indian J Ophthalmol 2006; 54(1): 17-22.

7 Pinilla I, Ferreras A, Idoipe M, Sanchez-Cano AI, Perez-Garcia D, Herrera LX et al. Changes in frequency-doubling perimetry in patients with type I diabetes prior to retinopathy. Biomed Res Int 2013; 2013: 341269.

8 Gualtieri M, Bandeira M, Hamer RD, Damico FM, Moura AL, Ventura DF. Contrast sensitivity mediated by inferred magnoand parvocellular pathways in type 2 diabetics with and without nonproliferative retinopathy. Invest Ophthalmol Vis Sci 2011; 52(2): 1151-1155.

9 Dosso AA, Bonvin ER, Morel Y, Golay A, Assal JP, Leuenberger PM. Risk factors associated with contrast sensitivity loss in diabetic patients. Graefes Arch Clin Exp Ophthalmol 1996; 234(5): 300-305.

10 Verma A, Rani PK, Raman R, Pal SS, Laxmi G, Gupta M et al. Is neuronal dysfunction an early sign of diabetic 
retinopathy? Microperimetry and spectral domain optical coherence tomography (SD-OCT) study in individuals with diabetes, but no diabetic retinopathy. Eye (Lond) 2009; 23(9): 1824-1830.

11 Peng PH, Lin HS, Lin S. Nerve fibre layer thinning in patients with preclinical retinopathy. Can J Ophthalmol 2009; 44(4): 417-422.

12 Cabrera DeBuc D, Somfai GM. Early detection of retinal thickness changes in diabetes using optical coherence tomography. Med Sci Monit 2010; 16(3): MT15-MT21.

13 van Dijk HW, Kok PH, Garvin M, Sonka M, Devries JH, Michels RP et al. Selective loss of inner retinal layer thickness in type 1 diabetic patients with minimal diabetic retinopathy. Invest Ophthalmol Vis Sci 2009; 50(7): 3404-3409.

14 van Dijk HW, Verbraak FD, Kok PH, Garvin MK, Sonka M, Lee $\mathrm{K}$ et al. Decreased retinal ganglion cell layer thickness in patients with type 1 diabetes. Invest Ophthalmol Vis Sci 2010; 51(7): 3660-3665.

15 The Diabetes Control and Complications Trial Research Group. The effect of intensive treatment of diabetes on the development and progression of long-term complications in insulin-dependent diabetes mellitus. N Engl J Med 1993; 329 (14):977-986.

16 UK Prospective Diabetes Study (UKPDS) Group. Intensive blood-glucose control with sulphonylureas or insulin compared with conventional treatment and risk of complications in patients with type 2 diabetes (UKPDS 33). Lancet 1998; 352 (9131):837-853.

17 Brownlee M, Hirsch IB. Glycemic variability: a hemoglobin A1c-independent risk factor for diabetic complications. JAMA 2006; 295(14): 1707-1708.

18 Lachin JM, Genuth S, Nathan DM, Zinman B, Rutledge BN. Effect of glycemic exposure on the risk of microvascular complications in the diabetes control and complications trial —revisited. Diabetes 2008; 57(4): 995-1001.

19 Monnier L, Mas E, Ginet C, Michel F, Villon L, Cristol JP et al. Activation of oxidative stress by acute glucose fluctuations compared with sustained chronic hyperglycemia in patients with type 2 diabetes. JAMA 2006; 295(14): 1681-1687.

20 Trence DL, Hirsch IB. Motherhood, apple pie, hemoglobin A (1C), and the DCCT. Endocr Pract 2012; 18(1): 78-84.

21 Kilpatrick ES, Rigby AS, Atkin SL. A1C variability and the risk of microvascular complications in type 1 diabetes: data from the Diabetes Control and Complications Trial. Diabetes Care 2008; 31(11): 2198-2202.

22 Quagliaro L, Piconi L, Assaloni R, Martinelli L, Motz E, Ceriello A. Intermittent high glucose enhances apoptosis related to oxidative stress in human umbilical vein endothelial cells: the role of protein kinase $\mathrm{C}$ and $\mathrm{NAD}(\mathrm{P}) \mathrm{H}-$ oxidase activation. Diabetes 2003; 52(11): 2795-2804.

23 Jaiswal M, McKeon K, Comment N, Henderson J, Swanson S, Plunkett $\mathrm{C}$ et al. Association between impaired cardiovascular autonomic function and hypoglycemia in patients with type 1 diabetes. Diabetes Care 2014; 37(9): 2616-2621.

24 Di Flaviani A, Picconi F, Di Stefano P, Giordani I, Malandrucco I, Maggio P et al. Impact of glycemic and blood pressure variability on surrogate measures of cardiovascular outcomes in type 2 diabetic patients. Diabetes Care 2011; 34(7): 1605-1609.

25 American Diabetes A. Executive summary: standards of medical care in diabetes-2014. Diabetes Care 2014; 37(Suppl 1): S5-S13.

26 Chiu SJ, Li XT, Nicholas P, Toth CA, Izatt JA, Farsiu S. Automatic segmentation of seven retinal layers in SDOCT images congruent with expert manual segmentation. Opt Express 2010; 18(18): 19413-19428.

27 Boynton GE, Stem MS, Kwark L, Jackson GR, Farsiu S, Gardner TW. Multimodal characterization of proliferative diabetic retinopathy reveals alterations in outer retinal function and structure. Ophthalmology 2015; 122(5): 957-967.

28 Wilkinson CP, Ferris FL III, Klein RE, Lee PP, Agardh CD, Davis $\mathrm{M}$ et al. Proposed international clinical diabetic retinopathy and diabetic macular edema disease severity scales. Ophthalmology 2003; 110(9): 1677-1682.

29 Kovatchev BP, Cox DJ, Gonder-Frederick LA, Young-Hyman D, Schlundt D, Clarke W. Assessment of risk for severe hypoglycemia among adults with IDDM: validation of the low blood glucose index. Diabetes Care 1998; 21(11): 1870-1875.

30 Scott IU, Jackson GR, Quillen DA, Larsen M, Klein R, Liao J et al. Effect of doxycycline vs placebo on retinal function and diabetic retinopathy progression in patients with severe nonproliferative or non-high-risk proliferative diabetic retinopathy: a randomized clinical trial. JAMA Ophthalmol 2014; 132(5): 535-543.

31 van Dijk HW, Verbraak FD, Kok PH, Stehouwer M, Garvin MK, Sonka M et al. Early neurodegeneration in the retina of type 2 diabetic patients. Invest Ophthalmol Vis Sci 2012; 53(6): 2715-2719.

32 Dhamdhere KP, Bearse Jr MA, Harrison W, Barez S, Schneck ME, Adams AJ. Associations between local retinal thickness and function in early diabetes. Invest Ophthalmol Vis Sci 2012; 53(10): 6122-6128.

33 Kashani AH, Zimmer-Galler IE, Shah SM, Dustin L, Do DV, Eliott D et al. Retinal thickness analysis by race, gender, and age using Stratus OCT. Am J Ophthalmol 2010; 149(3): 496-502 e1.

34 Bressler NM, Edwards AR, Antoszyk AN, Beck RW, Browning DJ, Ciardella AP et al. Retinal thickness on stratus optical coherence tomography in people with diabetes and minimal or no diabetic retinopathy. Am J Ophthalmol 2008; 145(5): 894-901.

35 Biallosterski C, van Velthoven ME, Michels RP, Schlingemann RO, DeVries JH, Verbraak FD. Decreased optical coherence tomography-measured pericentral retinal thickness in patients with diabetes mellitus type 1 with minimal diabetic retinopathy. Br J Ophthalmol 2007; 91(9): 1135-1138.

36 Holfort SK, Norgaard K, Jackson GR, Hommel E, Madsbad S, Munch IC et al. Retinal function in relation to improved glycaemic control in type 1 diabetes. Diabetologia 2011; 54(7): 1853-1861. 